\title{
An organotypic slice culture model of chronic white matter injury with maturation arrest of oligodendrocyte progenitors
}

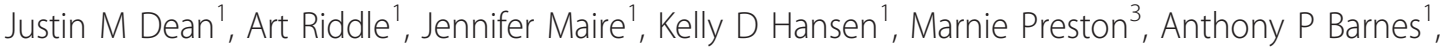 \\ Larry S Sherman ${ }^{3}$ and Stephen A Back ${ }^{1,2^{*}}$
}

\begin{abstract}
Background: CNS myelination disturbances commonly occur in chronic white matter lesions in neurodevelopmental and adult neurological disorders. Recent studies support that myelination failure can involve a disrupted cellular repair mechanism where oligodendrocyte (OL) progenitor cells (OPCs) proliferate in lesions with diffuse astrogliosis, but fail to fully differentiate to mature myelinating OLs. There are no in vitro models that reproduce these features of myelination failure.

Results: Forebrain coronal slices from postnatal day (P) 0.5/1 rat pups were cultured for 1, 5, or 9 days in vitro (DIV). Slices rapidly exhibited diffuse astrogliosis and accumulation of the extracellular matrix glycosaminoglycan hyaluronan (HA), an inhibitor of OPC differentiation and re-myelination. At 1 DIV $~ 1.5 \%$ of Olig2 ${ }^{+}$OLs displayed caspase- 3 activation, which increased to $\sim 11.5 \%$ by 9 DIV. At 1 DIV the density of PDGFR $\alpha^{+}$and PDGFRa $\alpha^{+} / \mathrm{Ki}_{67} 7^{+}$ OPCs were significantly elevated compared to 0 DIV $(P<0.01)$. Despite this proliferative response, at 9 DIV $\sim 60 \%$ of white matter OLs were late progenitors (preOLs), compared to $\sim 7 \%$ in the postnatal day 10 rat $(P<0.0001)$, consistent with preOL maturation arrest. Addition of HA to slices significantly decreased the density of $\mathrm{MBP}^{+}$OLs at 9 DIV compared to controls ( $217 \pm 16$ vs. $328 \pm 17$ cells $/ \mathrm{mm}^{2}$, respectively; $P=0.0003$ ), supporting an inhibitory role of HA in OL lineage progression in chronic lesions.
\end{abstract}

Conclusions: Diffuse white matter astrogliosis and early OPC proliferation with impaired OL maturation were reproduced in this model of myelination failure. This system may be used to define mechanisms of OPC maturation arrest and myelination failure related to astrogliosis and HA accumulation.

Keywords: white matter, oligodendrocyte, gliosis, astrocyte, hyaluronan, slice culture

\section{Introduction}

Disturbances in CNS myelination are a central feature of numerous neurodevelopmental and adult neurological disorders, and are widely recognized to occur in areas of reactive astrogliosis. Although myelination disturbances frequently involve oligodendrocyte (OL) degeneration [1-3], emerging evidence supports that OL progenitor cells (OPCs) exhibit a robust regenerative response to injury. In chronic white matter lesions, OPCs proliferate but fail to fully differentiate to mature myelinating OLs,

\footnotetext{
* Correspondence: backs@ohsu.edu

'Department of Pediatrics, Oregon Health \& Science University, 3181 SW

Sam Jackson Park Road, Portland, Oregon 97239, USA

Full list of author information is available at the end of the article
}

supporting the concept that failure to generate new myelin is related to arrest of oligodendrocyte maturation [4-6].

The mechanisms that mediate inhibition of $\mathrm{OL}$ maturation following CNS insults are largely unknown. Reactive astrogliosis is linked to OPC maturation arrest and remyelination failure in a number of conditions [7-9], and both Notch signaling and bone morphogenetic proteins induced during reactive gliosis have been implicated in these inhibitory processes $[10,11]$. Release of hyaluronan (HA) by reactive astrocytes also appears to be an important regulator of CNS myelination [12], and HA can arrest OPC maturation both in vitro and in vivo $[13,14]$. HA is a non-sulfated, protein-free

\section{() Biomed Central}


glycosaminoglycan that forms an integral part of the extracellular matrix. In the CNS, HA is predominantly synthesized by astrocytes, and can accumulate in areas of chronic astrocytosis and myelination disturbance [15]. $\mathrm{HA}$ and its receptor CD44 are robustly expressed in white matter lesions with diffuse astrogliosis, consistent with the response observed in demyelinating lesions, traumatic spinal cord injury, vascular brain injury associated with dementia, and ischemic lesions in adult humans and rodents $[14,16,17]$. The molecular mechanisms by which HA inhibits OL maturation are largely unknown, and as yet, there are no well-established in vitro models that reproduce the major features of chronic white matter lesions.

Herein, we developed a slice culture model of reactive astrogliosis that exhibited accumulation of HA in the white matter, with associated OPC proliferation but impaired maturation. Addition of HA to this system further impaired OPC maturation, providing support for an inhibitory role of HA in OL lineage progression. This chronic white matter injury model thus provides a novel system to define mechanisms of myelination failure related to astrogliosis and disturbances in oligodendrocyte maturation.

\section{Results}

Organotypic slice cultures display progressive diffuse astrogliosis and HA accumulation

Intact whole coronal forebrain slices containing white matter and overlying cortex were cultured from postnatal day 0.5/1 (P0.5/1) rats. To investigate the glial injury response in this slice culture model, we analyzed immunohistochemical expression of GFAP (astrocytes) and Iba1 (microglia/macrophages) in the white matter at 0 , 1,5 , and 9 days in vitro (DIV). At 0 DIV (i.e., P0.5/ 1 rat brain with no culture), there was negligible expression of GFAP in the white matter (Figure 1A), and low expression of Iba1 (Figure 1E) in cells that exhibited a resting morphology (Figure $1 \mathrm{E}$ inset). Compared to 0 DIV (Figure 1A), there was a rapid and progressive upregulation of GFAP expression in astrocytes (Figure 1BD) and Iba1 in microglia/macrophages (Figure 1E-H). Both cell types exhibited a reactive morphology (GFAP ${ }^{+}$ cells, Figure 1D inset; Iba1 ${ }^{+}$cells, Figure $1 \mathrm{H}$ inset). Overall, these data supported a pattern of progressive reactive gliosis.

Next, we detected HA expression with a biotinylated hyaluronan (HA) binding protein (bHABP). At 0 DIV (Figure 1I), there was low HA expression in the white matter, followed by a progressive increase from 1 DIV to 9 DIV (Figure 1J-L). Elevated HA was observed predominantly in areas of gliosis (Figure $1 \mathrm{M}-\mathrm{O}$ ), with a characteristic pericellular pattern of expression (Figure $1 \mathrm{P})$, consistent with previous observations [14]. Hence, chronic slice cultures displayed diffuse astrogliosis and HA accumulation similar to that observed in chronic cerebral whiter matter lesions in vivo.

\section{Oligodendrocyte (OL) survival and progenitor responses in white matter of organotypic slice cultures}

To investigate survival of the total OL lineage in this slice culture model, we examined the density of cleaved caspase-3 (CC3)-positive cells $\left(\mathrm{Olig} 2^{+}\right)$in the white matter at 1 DIV, 5 DIV, and 9 DIV (Figure 2A, B). Approximately $1.5 \%$ of all Olig2 ${ }^{+}$cells were positive for CC3 at 1 DIV. There was a progressive but non-significant increase to $\sim 11.5 \%$ by 9 DIV.

To examine the response of OPCs in the white matter of this slice culture model, we examined the density of PDGFR $\alpha^{+}$OPCs and their co-localization with the proliferation marker Ki67 at 0 DIV, 1 DIV, 5 DIV and 9 DIV (Figure 2C). At 1 DIV, there was a significant increase in the density of both PDGFR $\alpha^{+}$cells and PDGFR $\alpha^{+} / \mathrm{Ki} 67^{+}$ double-labeled cells compared to 0 DIV (ANCOVA + Tukey's test; $P<0.01$, for both), consistent with OPC proliferation. By 5 DIV and 9 DIV the density of both PDGFR $\alpha^{+}$cells and PDGFR $\alpha^{+} / \mathrm{Ki} 67^{+}$double-labeled cells returned to 0 DIV levels. With increasing time in culture, PDGFR $\alpha^{+}$OPCs exhibited a reactive-type of morphology, with increased cell body size and process thickness, as well as more extensive process arborization and complexity (Figure 2D-F). Hence, this slice culture model was associated with an acute phase of rapid OPC proliferation resulting in a net expansion in the OPC pool, followed by a delayed phase of degeneration.

\section{Delayed OPC maturation in white matter of organotypic slice cultures}

To determine the timing of OPC maturation, we quantified the relative percentages of late oligodendrocyte precursors (preOLs; $\mathrm{O}^{+} / \mathrm{O}^{-}$) and immature oligodendrocytes (immature OLs; $\mathrm{O}^{+} / \mathrm{O}^{+}$) in slice cultures at 9 DIV compared to the normal rat brain at an equivalent postnatal age (P10). Rat brains at P10 only expressed $7 \pm 2 \%$ preOLs in the white matter (Figure $3 \mathrm{~A}, \mathrm{E})$. Most $\mathrm{O}^{+} / \mathrm{O}^{+}$immature OLs displayed a reduced process arbor and extensive early myelination (Figure 3C). By contrast, there were $60 \pm 1 \%$ preOLs in the white matter in this slice culture model at 9 DIV (Figure 3B, E; $P<0.0001$ ). Interestingly, both preOLs and immature OLs displayed a reactive morphology, with a hypertrophic cell body and an extensive arbor of processes (Figure 3D) relative to normal brain at P10 (Figure $3 \mathrm{C}$ ). Some $\mathrm{O}^{+} / \mathrm{O}^{+}$OLs showed a highly branched morphology consistent with mature OLs (Figure $3 \mathrm{~F}$ ). Hence, the maturation of OPCs in this slice model was markedly delayed relative to the normal rat brain, with arrested OL maturation at the preOL stage. 


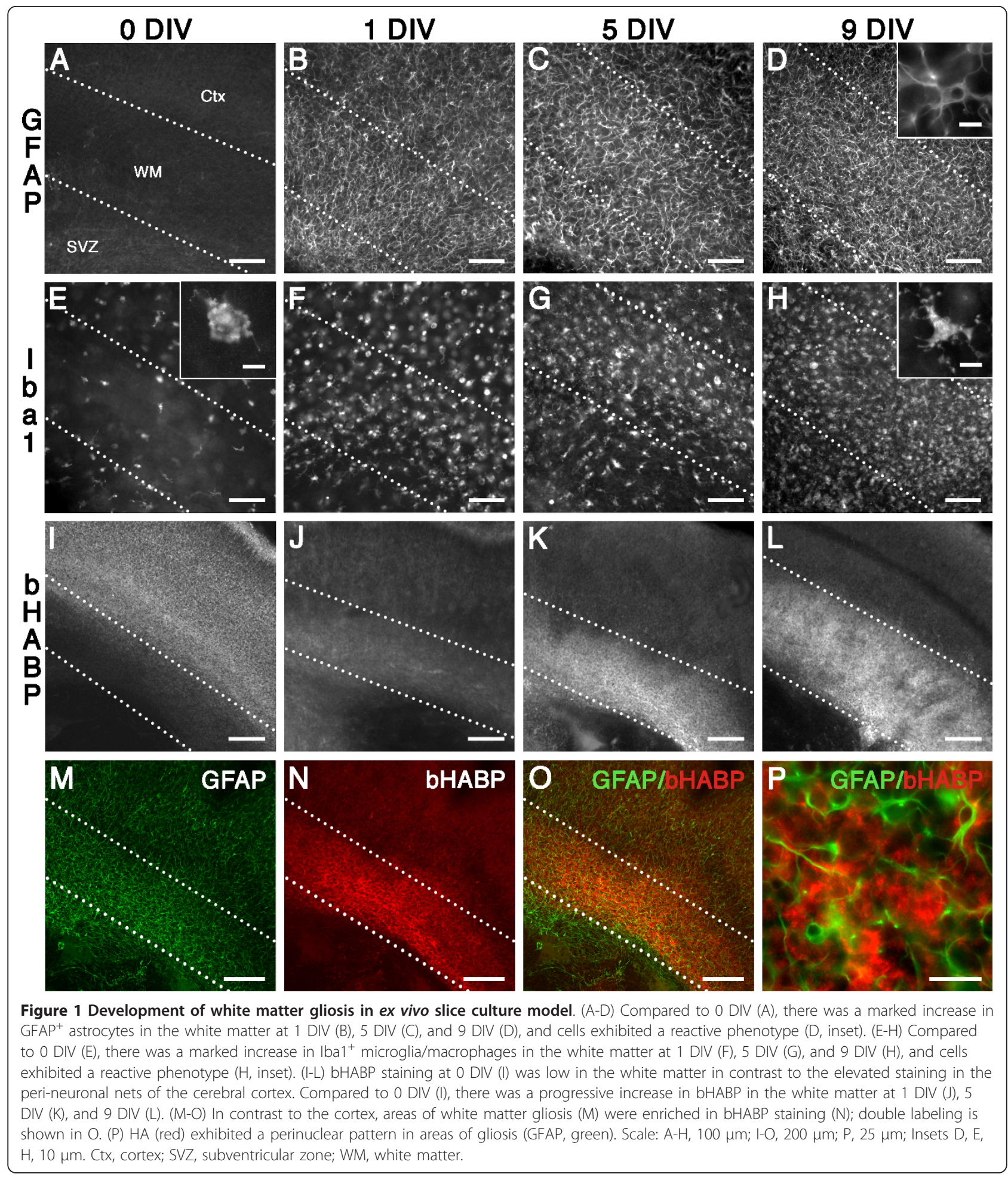

Timing of myelin onset in white matter of organotypic slice cultures

We examined the immunohistochemical expression of MBP in the white matter at 1 DIV, 5 DIV, 9 DIV, and 13 DIV. At 1 DIV there were no $\mathrm{MBP}^{+}$cells in the slice cultures (Figure 4A). By 5 DIV a low number of $\mathrm{MBP}^{+}$ mature OLs were observed (Figure 4B). By 9 DIV there was a marked increase in expression of individual $\mathrm{MBP}^{+}$ cells (Figure 4C). The $\mathrm{MBP}^{+}$cells at 9 DIV were highly branched but largely occupied individual domains, and 

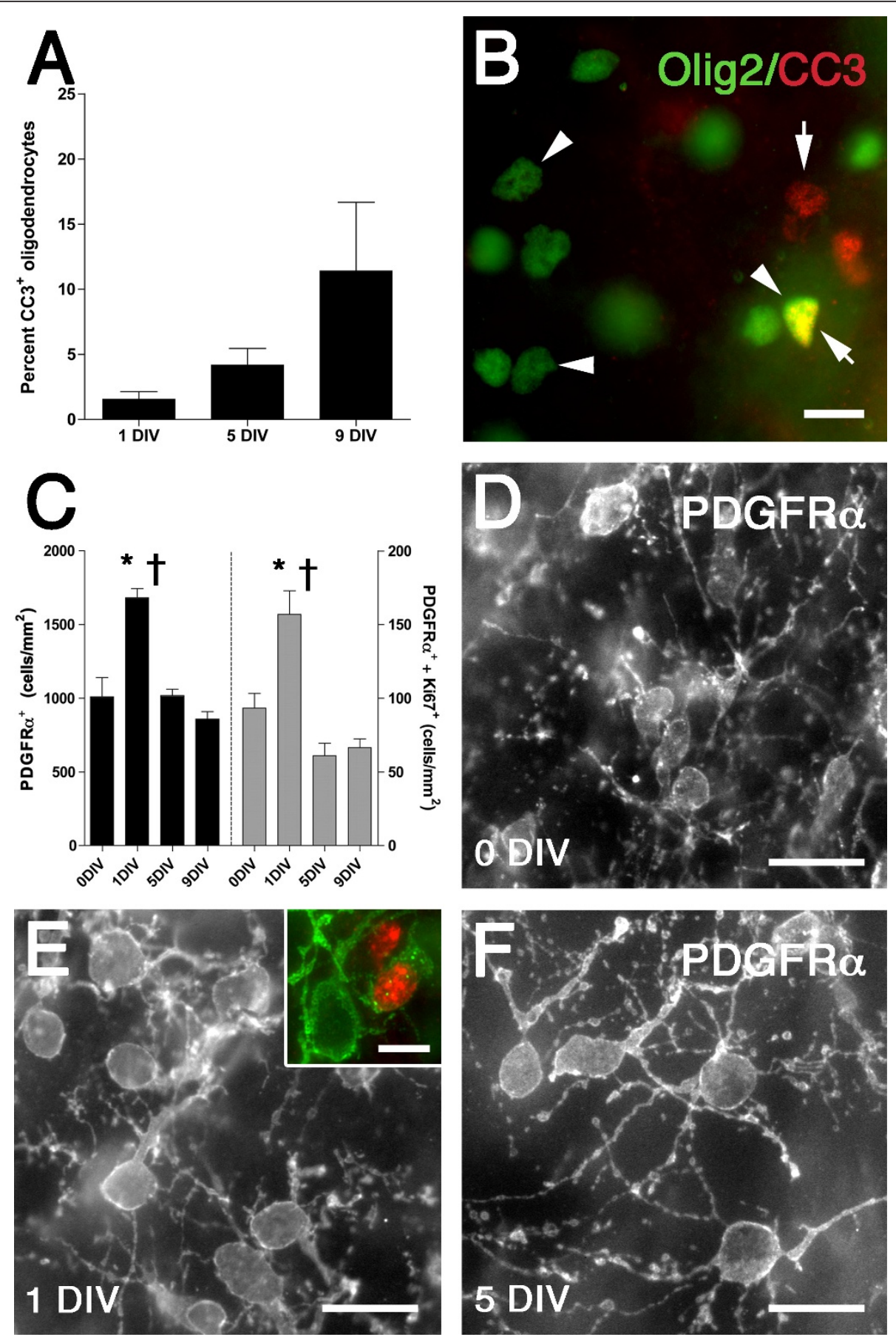

Figure 2 Oligodendrocyte $(\mathrm{OL})$ survival and oligodendrocyte progenitor proliferation in the white matter of ex vivo slice culture model. (A) Percentage of total OL lineage cells in the white matter that labeled with cleaved caspase- $3^{+}$(CC 3$)$. Data are mean \pm SEM ( $n=3-4$ slices per time point). (B) Example of $\mathrm{CC}^{+}$(red, arrows) and Olig2 ${ }^{+}$(green, arrowheads) cells (co-localized cells are shown in yellow). (C) Density of PDGFR $\alpha^{+}$(black bars, left axis) and co-localized PDGFR $\alpha^{+} / \mathrm{Ki}_{6} 7^{+} \mathrm{OPCs}$ in the white matter. Data are mean \pm SEM ( $\mathrm{n}=6$ slices per time point from two independent experiments). ${ }^{*} P<0.01$ (ANOVA followed by Tukey's multiple comparison test; uncorrected data). $+P<0.01$ (ANCOVA followed by Tukey's multiple comparison test; data corrected for atrophy). (D-F) PDGFR $\alpha^{+}$OPCs in the white matter at 0 DIV (D), 1 DIV (E) and 5 DIV (F). The inset in E shows a PDGFRa ${ }^{+}$(green)/Ki67 ${ }^{+}$(red) double labeled OPC. Scale: B, D-F, $20 \mu \mathrm{m}$; E inset, $10 \mu \mathrm{m}$. 

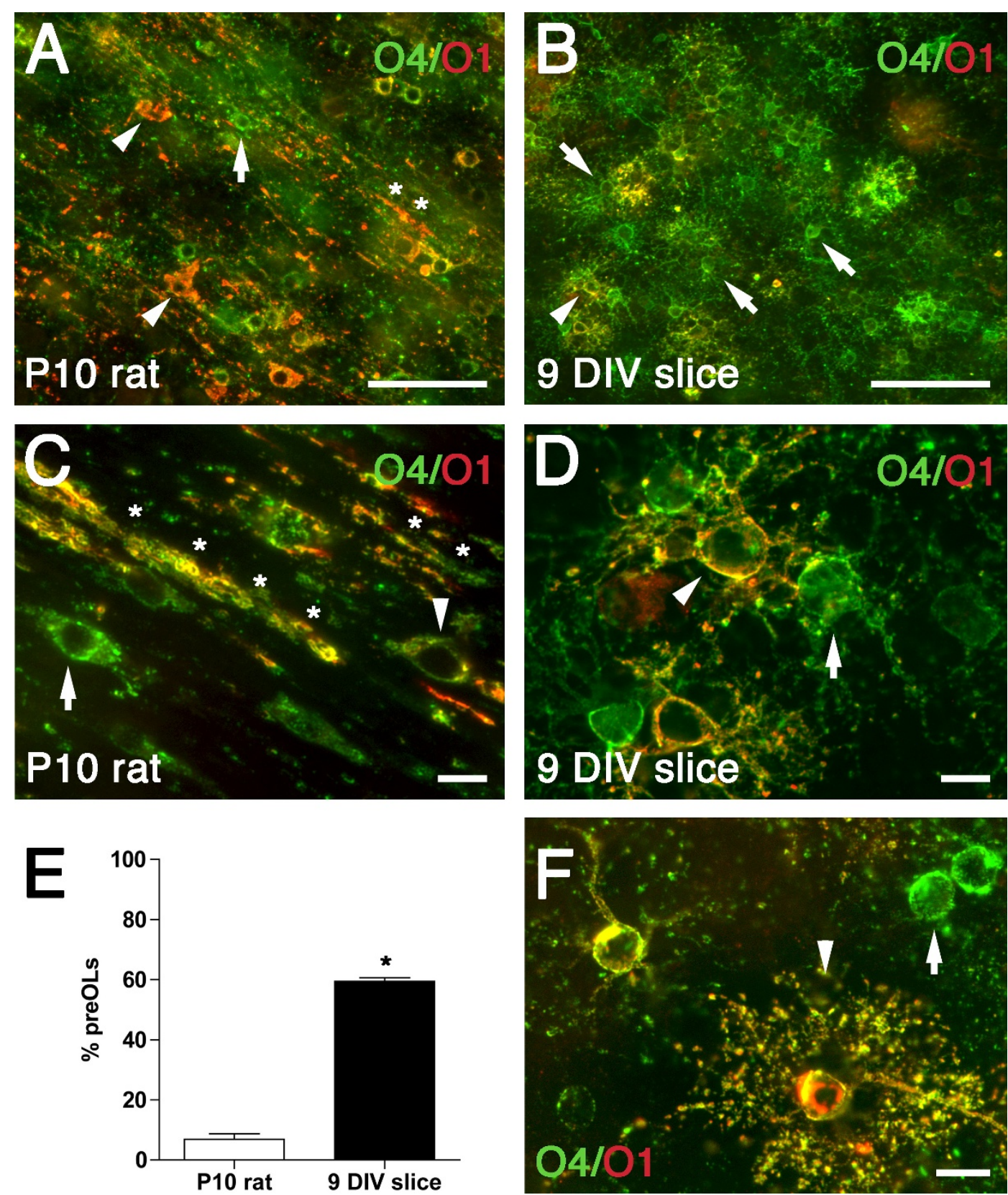

Figure 3 Oligodendrocyte maturation is delayed in the white matter of the ex vivo slice culture model compared to normal white matter development in vivo. Representative photomicrographs of preOLs (green; arrows) and immature OLs (yellow; arrowheads) doublelabeled with $\mathrm{O} 4$ (green) and $\mathrm{O} 1$ (red) antibodies in the P10 rat ( $\mathrm{A}, \mathrm{C})$ and the white matter of the 9 DIV slice (B, D, F). (A, C) The P10 rat white matter contained numerous immature OLs (arrowheads) and myelin sheaths (asterisk). (B, D) Slice cultures contained predominantly preOLs (green; arrows) with occasional immature OLs (yellow; arrowheads). Both preOLs and immature OLs in the slice cultures showed a reactive morphology, with extensive process extension and cytoplasmic swelling when compared with normal brain at P10 (C). (E)

Percentage of preOLs in the white matter of P10 rat (white bar) vs. organotypic slice culture at 9 DIV (Black bar). Data are mean \pm SEM ( 9 DIV slice data: $\mathrm{n}=11$ slices from three independent experiments; $\mathrm{P} 10$ rat data: $\mathrm{n}=6$ animals). ${ }^{*} P<0.0001$ (unpaired two-way t-test). (F) Some $\mathrm{O}^{+} / \mathrm{O}^{+} \mathrm{OLs}$ in slice culture showed a highly branched morphology (arrowhead) suggestive of a mature OL; arrow indicates a preOL. Scale: $A, B, 50 \mu m ; C, D, F, 10 \mu m$. 

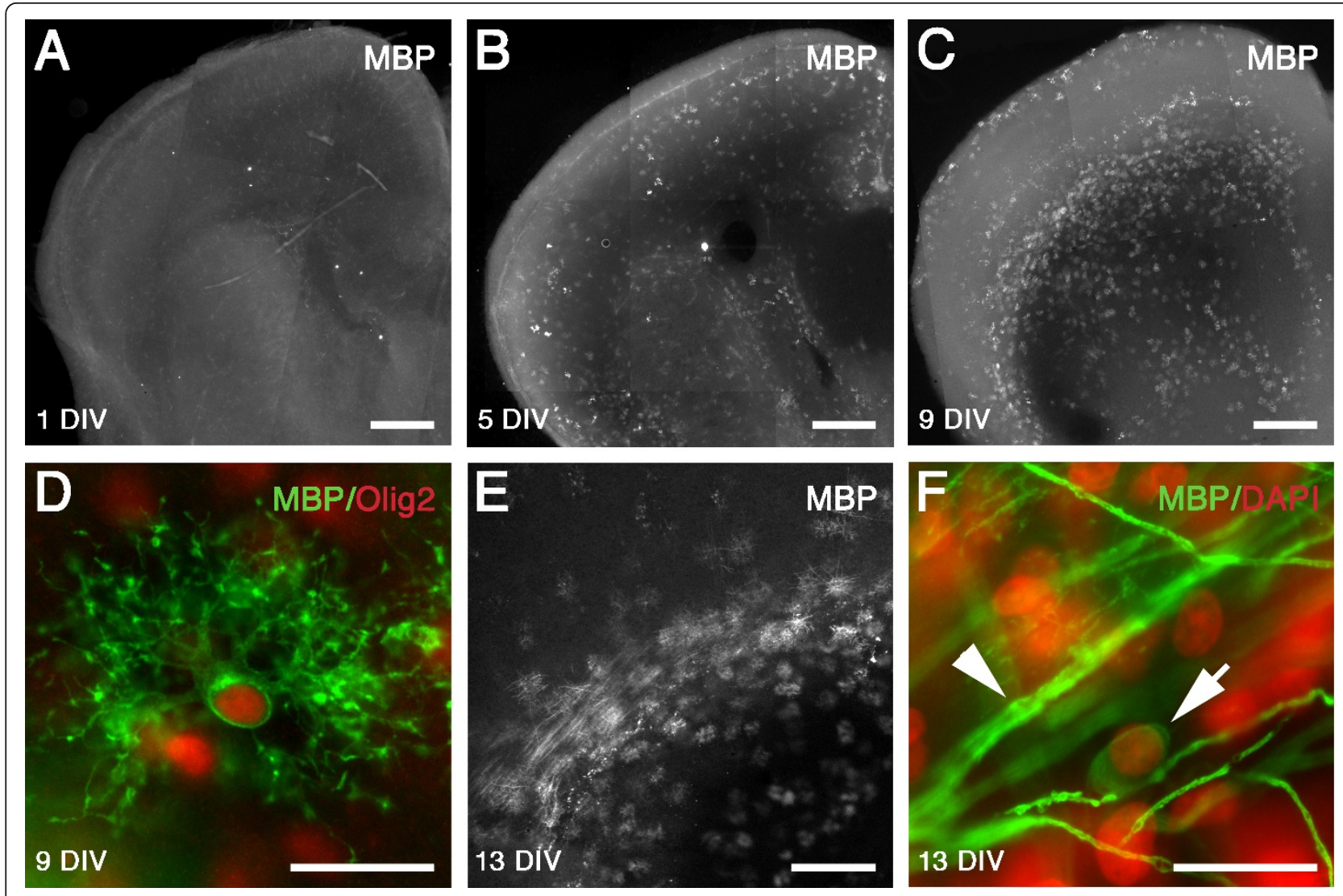

Figure 4 Timing of myelin onset in white matter of ex vivo slice culture model. MBP expression at 1 DIV (A), 5 DIV (B), and 9 DIV (C) in white matter of organotypic slice cultures. There was a progressive increase in MBP expression in the white matter with time in culture. (D) At 9 DIV, robust expression of individual MBP positive cells was observed, which were co-localized with the oligodendrocyte nuclear transcription factor Olig2 (MBP: green, Olig2: red). (E) Evidence of onset of myelination in the white matter at 13 DIV. (F) Oligodendrocyte (arrow; DAPI: shown as pseudo-color in red) showing wrapping of axons stained with MBP (green; arrowhead). Scale: A-C, $500 \mu \mathrm{m} ; \mathrm{D}, \mathrm{F}, 20 \mu \mathrm{m}$; E, $250 \mu \mathrm{m}$.

co-localized with the nuclear oligodendrocyte marker Olig2 (Figure 4D). At 13 DIV a small number of $\mathrm{MBP}^{+}$ cells appeared to be initiating myelination (Figure 4E, F). Despite the presence of a subpopulation of mature OLs in the white matter, there was thus a pronounced reduction in myelination compared to the normal rat brain at P10 (Figure 3C) or P14 [18,19].

\section{Exogenous hyaluronan impairs oligodendrocyte} maturation in white matter of organotypic slice cultures We added high molecular weight HA to this slice culture system to examine its role in regulating oligodendrocyte maturation. At 9 DIV there was a significant decrease in the density of $\mathrm{MBP}^{+}$oligodendrocytes in the white matter in slices treated with HA compared to those treated with PBS $(\sim 34 \% ; 217 \pm 16$ vs. $328 \pm 17$ cells $/ \mathrm{mm}^{2}$, respectively; $P=0.0003$; Figure $5 \mathrm{~A}-\mathrm{C}$ ). Hence, exogenous HA further reduced the level of OL maturation relative to that in untreated chronic slices where OL maturation was already markedly lower than equivalent age uninjured control animals.

\section{Discussion}

The normal regulation of oligodendrocyte (OL) maturation and myelination in the CNS is critical for normal vertebrate function, as well as to promote recovery following white matter injury. Dissociated OL cultures have provided important information on many aspects of these processes. Nevertheless, there are no well-established in vitro models that accurately model the chronic gliotic lesions often observed in disorders with myelination failure. There is now increasing interest in the use of organotypic slice cultures for studies of OL biology and myelination due to retention of multi-cellular interactions and the ease of manipulation [20-22]. However, we found that organotypic slice cultures display features consistent with a chronic white matter injury in vivo, which included white matter gliosis, cell death, OPC proliferative and reactive responses and arrested OL lineage maturation. Hence, when employing a chronic slice culture model for studies of normal oligodendroglial biology or myelination, these chronic injury responses should be considered. 
morphology, delayed OL degeneration, and impaired maturation of preOLs to OLs. Delayed OPC maturation did not appear to be region-specific and cortical OPC maturation by 9 DIV also was delayed relative to the extensive myelination that occurs in vivo by postnatal day 10 . These in vitro responses are very similar to those first described in neonatal rodents following hypoxia-ischemia, where despite extensive OL degeneration, there was a rapid proliferative response of OPCs, subsequent failure of preOLs to mature in chronic astrogliotic lesions, and persistent myelination deficits [4]. A role for OL maturation arrest in developmental myelination disturbance is supported by more recent studies in preterm fetal sheep following hypoxia-ischemia [23] and in preterm human autopsy cases with chronic white matter injury (Buser et al., submitted). OPCs also accumulate but fail to mature to myelinating OLs in chronic demyelinated lesions in multiple sclerosis patients [24-26]. In a recent postmortem study of brains from human cases of age-related cognitive decline associated with vascular brain injury, a significant increase in the total pool of OLs was correlated with changes in MRI-defined diffusion characteristics consistent with white matter myelin deficits [27].

Our slice culture system exhibited a progressive increase in expression of HA in the white matter with time in culture, while addition of exogenous HA further inhibited OL maturation. These data suggest a role for endogenously released HA in mediating the impairment of OL maturation observed in this system, and support previous studies where addition of HA reversibly impaired maturation of cultured OPCs and inhibited remyelination after lysolethicin-induced white matter demyelination $[13,14]$. Accumulation of HA was also reported following traumatic spinal cord injury [16] and middle cerebral artery occlusion [28] in adult rats, and in stroke-affected brain regions in adult humans [29], although myelination deficits were not examined. The increase in white matter HA in the slice cultures was likely a response to the reactive astrogliosis, because in the CNS, HA is predominantly produced by astrocytes [30]; however, we cannot discount a role of other CNS glia. The reactive gliosis observed in this system was likely a response to neuronal and glial degeneration secondary to generation of the tissue slices.

\section{Conclusions}

This model provides a novel system to define mechanisms that regulate disturbances in oligodendrocyte maturation and myelination failure related to chronic CNS astrogliosis. Future studies will utilize this model to determine the signaling pathways by which HA regulates oligodendrocyte development in the setting of chronic white matter injury.
Similar to recent in vivo studies examining the chronic OL response to white matter injury, we found rapid and progressive reactive astrocytosis and microglia/macrophage accumulation in the white matter of slice cultures, as well as an early proliferative response of OPCs. This expanded pool of OPCs exhibited a reactive 


\section{Methods}

\section{Postnatal brain slice preparation and culture}

All animal procedures were approved by the OHSU Institutional Animal Care and Use Committee (IACUC) according to the NIH Guide for the Care and Use of Laboratory Animals. Timed pregnant Sprague-Dawley (SD) rats were purchased from Charles River (Hollister, CA, USA). Whole forebrain coronal slices $(300 \mu \mathrm{m}$; collected at the level of the rostral corpus callosum and anterior septal nuclei; 3 adjacent slices from each brain) collected from postnatal day (P) 0.5/1 rat pups were used to prepare organotypic cultures according to a previous method [31], with modifications. Brains were embedded in $1.5 \%$ low melting point agar (Fischer Scientific, Fair Lawn, NJ, USA) and sectioned into sterile ice-cold complete Hank's balanced salt solution (HBSS, $\mathrm{Ca}^{2+} / \mathrm{Mg}^{2+}$ free [Invitrogen Co., Carlsbad, USA], supplemented with D-glucose [30 mM), HEPES buffer [2.5 $\mathrm{mM}], \mathrm{CaCl}_{2}[1 \mathrm{mM}], \mathrm{MgSO}_{4}[1 \mathrm{mM}], \mathrm{NaHCO}_{3}[4$ $\mathrm{mM}$ ], and $0.001 \%$ phenol red [Sigma-Aldrich Co., St. Louis, MO, USA]) using a VTS 1600 vibrating microtome (Leica Microsystems Inc., Buffalo Grove, USA). Isolated slices were transferred onto $0.4 \mu \mathrm{m}$ porous membrane cell culture inserts (Becton Dickinson, Franklin Lakes, NJ, USA) that were pre-coated with laminin/ poly-D-lysine (Sigma-Aldrich Co.), and cultured in slice culture media (Basal Medium Eagle [Invitrogen Co.], supplemented with complete HBSS [25\% v/v], D-glucose [27 $\mathrm{mM}]$, penicillin $[100 \mathrm{U} / \mathrm{mL}]$, streptomycin $[100 \mathrm{U} /$ $\mathrm{mL}$ ], glutamine [1 mM; Sigma-Aldrich Co.], and 5\% horse serum [New Zealand origin, heat inactivated; Invitrogen Co.]). Slices were incubated at $37^{\circ} \mathrm{C} / 5 \% \mathrm{CO}_{2}$, and the growth medium changed daily. In pilot experiments we determined that $5 \%$ serum resulted in optimal acute survival of the slices when compared to $25 \%$ serum, as previously reported [21].

\section{Time course experiments}

Slices were collected at $1,5,9$, or 13 days in vitro (DIV), fixed in $4 \%$ paraformaldehyde (PFA; $0.1 \mathrm{M}$ phosphate buffered saline [PBS]) for $1 \mathrm{~h}$ at RT, and washed thoroughly in PBS prior to immunohistochemical staining. As controls, slices were fixed immediately after cutting (0 DIV; i.e., no culture).

\section{Hyaluronan experiments}

High molecular weight hyaluronan $\left(1.59 \times 10^{6} \mathrm{Da}\right.$; Seikagaku Co., Tokyo, Japan) was dissolved in sterile PBS $(5 \mathrm{mg} / \mathrm{mL})$, and then added daily to fresh slice growth medium (final concentration, $100 \mu \mathrm{g} / \mathrm{mL}$ ) from 0 DIV until 9 DIV [14]. Slices were then processed for immunohistochemistry as described.

\section{Immunohistochemistry}

Single- or double-labeling immunofluorescence was performed on sections in 24-well plates. Antibodies and dilutions are shown in Table 1. For PDGFR $\alpha / \mathrm{Ki} 67 \mathrm{dou}-$ ble labeling and $\mathrm{O} 4$ and $\mathrm{O} 1$ antibody double labeling, sections were blocked for $1 \mathrm{~h}$ in PBS containing 5\% normal goat serum (NGS), and then with primary antibodies diluted in PBS/3\% NGS for $72 \mathrm{~h}$ at $4^{\circ} \mathrm{C}$ [4]. For all other antibodies, sections were incubated in $0.01 \mathrm{M}$ citrate buffer ( $\mathrm{pH} \mathrm{6.0)}$ ) at $85^{\circ} \mathrm{C}$ for $20 \mathrm{~min}$, left to cool for $20 \mathrm{~min}$, and then washed three times in PBS. Blocking and primary antibody incubation was performed as above, with the addition of $0.4 \%$ triton X-100 (SigmaAldrich Co.). For secondary detection, all sections were washed three times in PBS, and incubated with

\section{Table 1 Antibodies and markers}

\begin{tabular}{|c|c|c|c|c|}
\hline Antigen & Host & Marker & Dilution & Supplier \\
\hline $\begin{array}{l}\text { Biotinylated hyaluronan binding } \\
\text { protein (bHABP) }\end{array}$ & Bovine & Hyaluronan & $1: 200$ & Associates of Cape Cod, Inc., East Falmouth, MA \\
\hline Cleaved caspase-3 (CC3) & Polyclonal Rabbit & Apoptotic cells & $1: 500$ & Cell Signaling Technology, Danvers, MA \\
\hline GFAP & Polyclonal Rabbit & Astrocytes & $1: 500$ & Dako North America, Inc., Carpinteria, CA \\
\hline Iba1 & Polyclonal rabbit & Microglia/macrophage & $1: 500$ & Wako Chemicals USA Inc., Richmond, VA \\
\hline Ki67 & $\begin{array}{l}\text { Monoclonal } \\
\text { mouse }\end{array}$ & Cell Cycle Activation & $1: 200$ & Novocastra, Buffalo Grove, IL \\
\hline MBP & Mouse & Mature OL & $1: 500$ & Covance, Princeton, NY \\
\hline$\overline{\mathrm{O}_{1}}$ & $\begin{array}{l}\text { Monoclonal } \\
\text { mouse lgM }\end{array}$ & Immature/mature OL & $1: 3000$ & $\begin{array}{l}\text { Dr. Rashmi Bansal (University of Connecticut Health } \\
\text { Center, Farmington, CT) }\end{array}$ \\
\hline Biotinylated $\mathrm{O}_{4}$ & $\begin{array}{l}\text { Monoclonal } \\
\text { mouse lgM }\end{array}$ & $\begin{array}{l}\text { Late OL progenitor/ } \\
\text { immature OL }\end{array}$ & $1: 500$ & Research Genetics, Huntsville, AL \\
\hline PDGFR $\alpha$ & Polyclonal rabbit & OL progenitor & $1: 1000$ & R\&D Systems, Minneapolis, MN \\
\hline Olig2 & Mouse & Oligodendrocyte (OL) & $1: 250$ & $\begin{array}{l}\text { Dr. John Alberta (Dana-Farber Cancer Institute, Boston, } \\
\text { MA }\end{array}$ \\
\hline
\end{tabular}


appropriate AlexaFluor fluorescent dye conjugated secondary antibodies (1:500, all raised in goat; Invitrogen Co.). Nuclei were counterstained with DAPI (Invitrogen Co.). No-primary-control studies for all antibodies exhibited no positive staining. Sections were mounted with Vectashield fluorescent mounting medium (Vector Laboratories, Inc., Burlingame, CA, USA).

\section{Cell quantification}

The white matter of cultured slices was analyzed using a Leica DMIRE2 inverted fluorescence microscope (Leica Microsystems Inc., Buffalo Grove, IL, USA) coupled to a Stereoinvestigator stereology system (MBF Bioscience, Williston, VT, USA). For each slice, the entire white matter (defined by DAPI-staining) region of interest (ROI) was traced at $5 \times$ magnification. Using the software to maintain white matter boundaries, cell counts were performed using the optical fractionator probe (Grid size, $300 \times 400 \mu \mathrm{m}$; Counting frame, $30 \times 30 \mu \mathrm{m}$; z-depth $20 \mu \mathrm{m}$ ) at $40 \times$ magnification in a minimum of 10 randomly selected white matter fields per slice. The slice thickness was also measured at each counting site. Cell density $\left(\mathrm{mm}^{2}\right)$ was calculated by the formula: [cell counts/(number of fields $\times$ counting frame area $\left.\left(\mathrm{mm}^{2}\right)\right)$ ].

\section{Statistics}

An unpaired two-tailed t-test was used to compare percentage OLs between normal rat brain and the slice cultures. One-way analysis of variance (ANOVA) followed by Tukey's multiple comparison test was used to assess changes in percentage $\mathrm{CC}^{+}$OLs in the white matter over time in culture. To determine the effect of slice atrophy on PDGFR $\alpha$ cell density measurements, we quantified mean white matter volume. For each slice, white matter volume was calculated from white matter ROI area $\times$ mean white matter thickness (determined from all count sites) as acquired during cell counting. One-way analysis of variance (ANOVA) followed by Tukey's multiple comparison test was first run to determine relative atrophy with time in culture. There was a significant overall effect of group $(P<0.01)$, with a significant decrease in white matter volume at 5 DIV and 9 DIV compared to other ages (white matter volume, $\mathrm{mm}^{3}$ : 0 DIV, $0.26 \mathrm{~mm}^{3}$; 1 DIV, $0.29 \mathrm{~mm}^{3}$; 5 DIV, 0.18 $\mathrm{mm}^{3}$; 9 DIV, $\left.0.18 \mathrm{~mm}^{3} ; P<0.05\right)$, which was attributed to a shrinkage of the white matter ROI area rather than in the z-plane of the slice (data not shown). Because of this reduction in white matter volume, we accounted for degree of atrophy by using white matter volume as a covariate when comparing cell density measurements between time in culture using a one-way analysis of covariance (ANCOVA) followed by Tukey's multiple comparison test. Cell density data were presented uncorrected for atrophy. A P-value less than 0.05 was considered statistically significant. All data are presented as mean \pm standard error of the mean (SEM), with significance indicated for both ANOVA and ANCOVA analyses (For figure $2,{ }^{*} P<0.01$ refers to analysis of uncorrected data by ANOVA; $+P<0.01$ refers to analysis of data corrected for slice atrophy by ANCOVA).

\section{Acknowledgements}

Supported by the National Institutes of Neurological Diseases and Stroke: 1RO1NS054044, R37NS045737-06S1/06S2 to SAB and 1F30NS066704 to AR, a Bugher Award from the American Heart Association (SAB) and the March of Dimes Birth Defects Foundation (SAB). LS was supported by NIH core grant RR00163 supporting the Oregon National Primate Research Center. JD was supported by a Heubner Family Developmental Neurobiology of Disease Fellowship. The Olig2 antibodies were a generous gift of Dr. John Alberta. AB was supported by $\mathrm{NIMH}$ grant $\mathrm{K} 01 \mathrm{MH} 08025$

\section{Author details}

Department of Pediatrics, Oregon Health \& Science University, 3181 SW Sam Jackson Park Road, Portland, Oregon 97239, USA. Department of Neurology, Oregon Health \& Science University, 3181 SW Sam Jackson Park Road, Portland, Oregon 97239, USA. ${ }^{3}$ Division of Neuroscience, Oregon National Primate Research Center, Oregon Health \& Science University, 505 NW 185th Ave, Portland, OR 97006, USA

\section{Authors' contributions}

JD designed the study, performed slice culture experiments, data collection, and statistical analyses, and drafted the manuscript with SB. AR and AB assisted in study design and model development. JM and $\mathrm{KH}$ performed slice culture immunohistochemistry and data collection. MP prepared HA and verified its quality, and assisted with HA labeling protocols. LS and SB conceived of the study, and participated in its design and coordination. All authors read and approved the final manuscript.

\section{Competing interests}

The authors declare that they have no competing interests.

Received: 11 May 2011 Accepted: 5 July 2011 Published: 5 July 2011

\section{References}

1. Wilke S, Thomas R, Allcock N, Fern R: Mechanism of acute ischemic injury of oligodendroglia in early myelinating white matter: the importance of astrocyte injury and glutamate release. J Neuropath Exp Neurol 2004, 63:872-881.

2. Back SA, Luo NL, Mallinson RA, O'Malley JP, Wallen LD, Frei B, Morrow JD, Petito CK, Roberts CT Jr, Murdoch GH, Montine TJ: Selective vulnerability of preterm white matter to oxidative damage defined by $\mathrm{F} 2-$ isoprostanes. Ann Neurol 2005, 58:108-120.

3. Back SA, Han BH, Luo NL, Chricton CA, Xanthoudakis S, Tam J, Arvin KL, Holtzman DM: Selective vulnerability of late oligodendrocyte progenitors to hypoxia-ischemia. J Neurosci 2002, 22:455-463.

4. Segovia KN, McClure M, Moravec M, Luo NL, Wan Y, Gong X, Riddle A, Craig A, Struve J, Sherman LS, Back SA: Arrested oligodendrocyte lineage maturation in chronic perinatal white matter injury. Ann Neurol 2008, 63:520-530.

5. Fancy SP, Kotter MR, Harrington EP, Huang JK, Zhao C, Rowitch DH, Franklin RJ: Overcoming remyelination failure in multiple sclerosis and other myelin disorders. Exp Neurol 2010, 225:18-23.

6. Huang Z, Liu J, Cheung PY, Chen C: Long-term cognitive impairment and myelination deficiency in a rat model of perinatal hypoxic-ischemic brain injury. Brain Res 2009, 1301:100-109.

7. Skripuletz T, Bussmann JH, Gudi V, Koutsoudaki PN, Pul R, MoharreghKhiabani D, Lindner M, Stangel M: Cerebellar cortical demyelination in the murine cuprizone model. Brain Pathol 2010, 20:301-312.

8. Anderson JM, Hampton DW, Patani R, Pryce G, Crowther RA, Reynolds R, Franklin RJ, Giovannoni G, Compston DA, Baker D, Spillantini MG, Chandran S: Abnormally phosphorylated tau is associated with neuronal and axonal loss in experimental autoimmune encephalomyelitis and multiple sclerosis. Brain 2008, 131:1736-1748. 
9. Keirstead HS, Nistor G, Bernal G, Totoiu M, Cloutier F, Sharp K, Steward O: Human embryonic stem cell-derived oligodendrocyte progenitor cell transplants remyelinate and restore locomotion after spinal cord injury. The J Neurosci 2005, 25:4694-4705.

10. John GR, Shankar SL, Shafit-Zagardo B, Massimi A, Lee SC, Raine CS, Brosnan CF: Multiple sclerosis: re-expression of a developmental pathway that restricts oligodendrocyte maturation. Nat Med 2002, 8:1115-1121.

11. Wang Y, Cheng X, He Q, Zheng Y, Kim DH, Whittemore SR, Cao QL: Astrocytes from the contused spinal cord inhibit oligodendrocyte differentiation of adult oligodendrocyte precursor cells by increasing the expression of bone morphogenetic proteins. J Neurosci 2011, 31:6053-6058.

12. Sherman $L S$, Back SA: A 'GAG' reflex prevents repair of the damaged CNS. Trends Neurosci 2008, 31:44-52.

13. Sloane JA, Batt C, Ma Y, Harris ZM, Trapp B, Vartanian T: Hyaluronan blocks oligodendrocyte progenitor maturation and remyelination through TLR2. Proc Natl Adac Sci USA 2010, 107:11555-11560.

14. Back SA, Tuohy TM, Chen H, Wallingford N, Craig A, Struve J, Luo NL, Banine F, Liu Y, Chang A, et al: Hyaluronan accumulates in demyelinated lesions and inhibits oligodendrocyte progenitor maturation. Nat Med 2005, 11:966-972.

15. Asher R, Perides G, Vanderhaeghen JJ, Bignami A: Extracellular matrix of central nervous system white matter: demonstration of a hyaluronateprotein complex. J Neurosci Res 1991, 28:410-421.

16. Struve J, Maher PC, Li YQ, Kinney S, Fehlings MG, Kuntz Ct, Sherman LS: Disruption of the hyaluronan-based extracellular matrix in spinal cord promotes astrocyte proliferation. Glia 2005, 52:16-24.

17. Wang $X, X u L$, Wang $H$, Zhan $Y$, Pure E, Feuerstein GZ: CD44 deficiency in mice protects brain from cerebral ischemia injury. J Neurochem 2002, 83:1172-1179

18. Craig A, Ling Luo N, Beardsley DJ, Wingate-Pearse N, Walker DW, Hohimer AR, Back SA: Quantitative analysis of perinatal rodent oligodendrocyte lineage progression and its correlation with human. Exp Neurol 2003, 181:231-240.

19. Dean JM, Moravec MD, Grafe M, Abend N, Ren J, Gong X, Volpe JJ, Jensen FE, Hohimer AR, Back SA: Strain-specific differences in perinatal rodent oligodendrocyte lineage and its correlation with human. Dev Neurosci.

20. Yang $Y$, Lewis $R$, Miller $\mathrm{RH}$ : Interactions between oligodendrocyte precursors control the onset of CNS myelination. Dev Biol 2011, 350:127-138.

21. Gadea A, Aguirre A, Haydar TF, Gallo V: Endothelin-1 regulates oligodendrocyte development. J Neurosci 2009, 29:10047-10062.

22. Mi S, Miller RH, Tang W, Lee $X$, Hu B, Wu W, Zhang Y, Shields CB, Miklasz S, Shea D, Mason J, Franklin RJ, Ji B, Shao Z, Chedotal A, Bernard F, Roulois A, $X u J$, Jung $V$, Pepinsky B: Promotion of central nervous system remyelination by induced differentiation of oligodendrocyte precursor cells. Ann Neurol 2009, 65:304-315.

23. Riddle A, Dean JM, Buser JR, Gong X, Maire J, Chen K, Ahmad T, Chen V, Nguyen T, Kroenke CD, Hohimer AR, Back SA: Histopathological correlates of MRI-defined chronic perinatal white matter injury. Ann Neurol 2011.

24. Wolswijk G: Oligodendrocyte precursor cells in the demyelinated multiple sclerosis spinal cord. Brain 2002, 125:338-349.

25. Kuhlmann T, Miron V, Cui Q, Wegner C, Antel J, Bruck W: Differentiation block of oligodendroglial progenitor cells as a cause for remyelination failure in chronic multiple sclerosis. Brain 2008, 131:1749-1758.

26. Kremer D, Aktas O, Hartung HP, Kury P: The complex world of oligodendroglial differentiation inhibitors. Ann Neurol 2011, 69:602-618.

27. Back SA, Kroenke CD, Sherman LS, Lawrence G, Gong X, Taber ER, Sonnen JA, Larson EB, Montine TJ: White matter lesions defined by diffusion tensor imaging in older adults. Ann Neurol 2011.

28. Al Qteishat A, Gaffney JJ, Krupinski J, Slevin M: Hyaluronan expression following middle cerebral artery occlusion in the rat. Neuroreport 2006, 17:1111-1114.

29. Al'Qteishat A, Gaffney J, Krupinski J, Rubio F, West D, Kumar S, Kumar P, Mitsios N, Slevin M: Changes in hyaluronan production and metabolism following ischaemic stroke in man. Brain 2006, 129:2158-2176.

30. Marret S, Delpech B, Delpech A, Asou H, Girard N, Courel MN, Chauzy C, Maingonnat $C$, Fessard C: Expression and effects of hyaluronan and of the hyaluronan-binding protein hyaluronectin in newborn rat brain glial cell cultures. J Neurochem 1994, 62:1285-1295.
31. Polleux F, Ghosh A: The slice overlay assay: a versatile tool to study the influence of extracellular signals on neuronal development. Sci STKE 2002, 2002:pl9.

doi:10.1186/1750-1326-6-46

Cite this article as: Dean et al:: An organotypic slice culture model of chronic white matter injury with maturation arrest of oligodendrocyte progenitors. Molecular Neurodegeneration 2011 6:46.

\section{Submit your next manuscript to BioMed Central and take full advantage of:}

- Convenient online submission

- Thorough peer review

- No space constraints or color figure charges

- Immediate publication on acceptance

- Inclusion in PubMed, CAS, Scopus and Google Scholar

- Research which is freely available for redistribution

Submit your manuscript at www.biomedcentral.com/submit
C Biomed Central 